\title{
AN ELEMENTARY PROOF OF STEINHAUS'S THEOREM
}

\section{KARL STROMBERG ${ }^{1}$}

The following theorem was proved by $\mathrm{H}$. Steinhaus [2] for the case that $G$ is the real line and $\lambda$ is Lebesgue measure. In the form stated here, it is due to André Weil [3, p. 50].

THEOREM. Let $G$ be a locally compact group with identity $e$ and a left Haar measure $\lambda$. If $A$ is a $\lambda$-measurable subset of $G$ such that $0<\lambda(A)<$ $\infty$, then the set $A A^{-1}=\left\{y x^{-1}: x, y \in A\right\}$ has $e$ in its interior.

The customary proof of this well-known theorem uses the convolution product of functions on $G$. Our proof, which does not seem to be widely known, is shorter and more elementary, even for the real line, than any that we have seen. All references of the form $(a . b)$ are to be found in [1].

Proof. Since $\lambda$ is regular, we may suppose that $A$ is compact (11.32) and we may choose an open set $U \supset A$ such that $\lambda(U)<2 \lambda(A)(11.22)$. Next choose a neighborhood $V$ of $e$ such that $V A \subset U$ (4.10). We complete the proof by showing that $V \subset A A^{-1}$. Let $v \in V$. Then $v A \cap A \neq \varnothing$ because otherwise, since $v A \cup A \subset U$, we would have $\lambda(U) \geqq \lambda(v A)+\lambda(A)=2 \lambda(A)$. Therefore, there exist $x, y \in A$ such that $v x=y$ and so $v=y x^{-1} \in A A^{-1}$.

\section{REFERENCES}

1. E. Hewitt and K. A. Ross, Abstract harmonic analysis. Vol. I: Structure of topological groups. Integration theory, group representations, Die Grundlehren der math. Wissenschaften, Band 115, Academic Press, New York; Springer-Verlag, Berlin, 1963. MR 28 \#158.

2. H. Steinhaus, Sur les distances des points des ensembles de mesure positive, Fund. Math. 1 (1920), 93-104.

3. A. Weil, L'intégration dans les groupes topologiques et ses applications, 2ième éd., Actualités Sci. Indust., no. 869, Hermann, Paris, 1951.

Department of Mathematics, Kansas State University, Manhattan, Kansas 66502

Received by the editors October $1,1971$.

AMS 1970 subject classifications. Primary 28A70; Secondary 43 A05.

Key words and phrases. Haar measure, Steinhaus theorem.

1 This work was supported by NSF Grant \#GP-12364.

(c) American Mathematical Society 1972 